$4.3 \%$ in 2009 and the prevalence of HIV in newborns was $23.3 \%$ in 2010. To date, no study has been done in the DRC, specifically Kinshasa, to explore the PMTCT national protocol.

Purpose The study aimed at evaluating compliance with the PMTCT national protocol in the selected PMTCT sites of Kinshasa Methodology A quantitative approach was employed in this study with a total of 76 health care providers, specifically nurses, and 179 records of HIV + women in 18 PMTCT sites selected in Kinshasa. A health care provider self-reporting questionnaire and review of the records of HIV + women were used for compliance assessment. Data collected was analysed using the SSPS package, Version 19.0 and MS Excel 2007.

Results This study found that nurses and HIV+ women were non-compliant (less than $80 \%$ ) with the PMTCT national protocol. The score of compliance/non-compliance decreased through the continuum of PMTCT care with a peak in labour and delivery for HIV + women. Some factors were associated with nurses and HIV+ women's compliance or non-compliance with PMTCT national protocol.

Conclusion The non-compliancy of nurses and HIV+ women found in this study goes beyond the improvement of compliance with PMTCT national protocol and necessitates full and sustainable integration of PMTCT in maternal, newborn and child health services.

\section{P6.051 COST-EFFECTIVENESS OF THE COMPLEX HIV PROGRAMME AMONG THE INJECTING DRUG USERS (IDU) IN THE CITY OF BARNAUL, ALTAI TERRITORY, RUSSIA}

doi:10.1136/sextrans-2013-051184.1204

E Demyanenko, N Vagaitseva. NGO "AntiAIDS-Siberia", Barnaul, Russian Federation

Background In 2011 the NGO "AntiAIDS-Siberia" estimated the cost-effectiveness of the complex HIV programme among the IDU in Barnaul.

Tasks:

- to assess the prevalence of HIV, HCV, related risk behaviour among the PP clients and comparison group;

- to assess and compare the cost of PP, ART and HCV treatment.

\section{Methods}

- descriptive and analytical method of epidemiological analysis of HIV sickness rate;

- bio-behavioural survey among the IDU in Barnaul, 2011;

- mathematical analysis of HIV treatment costs in compliance with the standards.

Results Different preventive components such as voluntary counselling and testing, social and medical support of the IDU, condom and injecting equipment distribution and safe skills building have been implemented in Barnaul. The average cost of HIV-prevention programme among the IDU in Barnaul is $\$ 35000$ per year. At the average 1500 IDU are covered by PP per year, so the average expenditure for PP is $\$ 23$ per 1 client. Average annual cost of ART per patient is $\$ 11356$, cost of HCV treatment (Pegasys + Ribavirin) $\$ 19000$.

According to the results of bio-behavioural survey 3 out of 150 PP clients are HIV positive (2\%), 121 (80.7\%) have HCV. In comparison group 31 out of 150 have HIV (20.7\%), 130 (86.7\%) have $\mathrm{HCV}$. If we extrapolate from the data to all IDU covered by PP in 2011, we can conclude that PP help to prevent 280 IDU from infecting HIV and HCV. It saves $\$ 3000000$ per year in the budget, which are needed to provide ART and HC treatment. At an average 1 HIV IDU infects 4 partners during a year. Prevention of 1120 cases saves $\$ 3000000$ per year in the budget which is needed to provide ART.
Conclusion Preventive programmes cost the government much lower than HIV and HCV treatment and can be considered rather effective.

\section{P6.052 INTEGRATING FAMILY PLANNING SERVICES INTO HIV COMPREHENSIVE CARE AND TREATMENT CLINICS: A CASE OF THE AIDS SUPPORT ORGANIZATION (TASO) TORORO}

doi:10.1136/sextrans-2013-051184.1205

S Okoboi, L Namwanje, R Oceng. The AIDS Support Organization, Tororo, Uganda

Issue: More than 1.4 million women in Uganda report wanting to delay pregnancy, space their children, or stop childbearing altogether but that they are not currently using any contraceptive method. In late 2010, TASO integrated family planning services into its routine comprehensive HIV care clinics with aim of making FP services accessible, prevention of unintended pregnancies and reduction of mother to child transmission.

Description The intervention consisted of continuous sensitization meetings with staff and clients to explain the new intervention and ask for support. TASO staff identified the integration of condoms, oral, emergency contraceptive pills, injectable contraceptives and inplants as a feasible beginning for on-site FP services. Clients interested in IUD and permanent methods were referred to Tororo Hospital's FP clinic. Developed plans for integration including, how to obtain commodities, and triplicate referrals system

Results Overall, the frequency of modern method use among female clients increased from $6 \%$ in Ist quarter to $28 \%$ 2nd quarter to $41 \% 3$ rd quarter and finally to $49 \%$ in 4 th quarter of 2011 . Condom use increased from 8\% to 80\%. A 2012 staff evaluation showed that the majority of providers (92\%) and clients (97\%) felt that adding FP did not affect the quality of providing care and treatment services, and that the level of integration was appropriate and costeffective, added the benefits of reducing unintended pregnancy, reducing mother-to-child transmission of HIV, improving ease in talking about sex and fertility desires with clients, strengthened staff skills. Follow-up of clients using FP services offered an additional opportunity for adherence counselling.

Next step: Incorporating family planning (FP) into HIV care and treatment services is a promising model for integrating FP and HIV services in clinics with existing strong systems to accommodate added services.

\section{P6.053 EVALUATION OF COST \& EFFICIENCY OF ALTERNATIVE SERVICE DELIVERY MODELS FOR PROVIDING STI SERVICES TO HIGH RISK INDIVIDUALS IN INDIA}

doi:10.1136/sextrans-2013-051184.1206

'D L Delhikar, ${ }^{2} \mathrm{~F}$ Homan, ${ }^{1} \mathrm{~A}$ Das. ${ }^{1} \mathrm{FH}$ 360, New Delhi, India; ${ }^{2} \mathrm{FHI} 360$, North Carolina, NC, United States

Background Avahan, a large-scale HIV prevention programme, provides STI services to over 300,000 high risk individuals (HRIs) in six states of India through six different service delivery models varying by provision site (programme operated vs. contract provider) and use of outreach activities. This study aims to determine the cost-efficiency of alternative STI service delivery models.

Methods Data from the period between January to June 2010 was collected retrospectively from a stratified random sample of 30 selected NGO/CBOs, providing STI services to HRIs. Service statistics were obtained from Avahan's Computerized Management Information System, and cost data from the NGO/CBOs financial records. Cost and outcome performance from the $\mathrm{NGO/CBOs} \mathrm{were}$ annualised and standardised to reflect a high risk group size of 1,000. Analysis was done from a service delivery perspective and did not 